\title{
Coronary intervention via novel distal radial artery approach
}

\author{
Łukasz Koziński ${ }^{1}$, Alicja Dąbrowska-Kugacka², Zbigniew Orzałkiewicz ${ }^{1}$ \\ 1Department of Cardiology, District Hospital, Chojnice, Poland \\ 2Department of Cardiology and Electrotherapy, Medical University of Gdansk, Gdansk, Poland
}

Adv Interv Cardiol 2019; 15, 1 (55): 123-124

DOI: https://doi.org/10.5114/aic.2019.83779

Vascular access through the puncturing of the femoral artery has been for many years the basic procedure for percutaneous diagnostic and therapeutic coronary interventions. This access, however, has been related to numerous and serious complications so other areas for a vascular approach have been investigated. In many clinical trials, the benefits of radial artery puncture have been proven and nowadays it is the preferred one. Nevertheless, this access is not free from complications, among which the most important is the post-procedural radial artery occlusion. Due to the increasing number of repeated coronary procedures, this complication makes it impossible to reuse the radial artery as vascular access. Recently there have been reports of the possibility of using the distal part of the radial artery in the area of the anatomical snuffbox [1]. The possibility of maintaining patency of the radial artery in the traditional (proximal) site, even despite occlusion of the distal part, is one of the advantages of this puncture site. Moreover, with this approach antegrade flow through the superficial palmar arch will be maintained in the case of thrombosis at the puncture site. An additional convenience is the superficial position of the radial artery on the osseous medium within the distal access, which enables precise compression and shortening of the hemostasis period. The risk of an arterio-venous fistula and nerve injuries in the place of the snuffbox should also be negligible. Left-sided access from the snuffbox enables ergonomic performance of the procedure by placing the left upper limb on the right groin of the patient, and in the case of the right-sided access the examined limb can be positioned in a more physiological position.

A 69-year-old man presented with unstable angina pectoris. Coronary intervention was performed via distal radial access. Despite a well-palpable pulse, ultrasonography (Figure $1 \mathrm{~A}$ ) was performed before the procedure, in order to ascertain that the vessel was sufficient to puncture. Routine local anesthesia using $1 \mathrm{ml}$ of $2 \%$ lidocaine was used. The right radial artery was punctured in the area of the anatomical snuffbox using a $20 \mathrm{G}$ metallic entry needle with the anterior wall modified Seldinger technique, and next a 0.038 " $45 \mathrm{~cm}$ long mini-guidewire was advanced through the needle.

A 6 Fr $10 \mathrm{~cm}$ long sheath (Radifocus Introducer, Terumo) was inserted (Figures 1 B-D), and then spasmolytic (verapamil $2.5 \mathrm{mg}$ ) and anticoagulant (unfractionated heparin $80 \mathrm{U} / \mathrm{kg}$ ) drugs were administered. The procedure was done using a $5 \mathrm{Fr}$ diagnostic catheter (Tiger, Terumo) and a 6 Fr guiding catheter (EBU 3.5, Medtronic) with everolimus-eluting stent implantation to the left circumflex artery. The operator promptly pulled out the sheath, and fixed a standard bandage compression for $4 \mathrm{~h}$ on the puncture site. The whole procedure and access site were uncomplicated and the radial artery remained patent.

The distal radial approach appears to be a feasible and safe alternative to traditional radial puncture [1-4], especially if its potential to reduce the incidence of proximal radial artery occlusion can be verified. The employment of catheters bigger than 6 Fr may be limited due to the reduction of the artery lumen in its distal part. Nevertheless, the use of vessel sheaths with smaller outer diameter (e.g. Glidesheath Slender, Terumo) or sheathless catheters may be helpful. According to the authors' experience both bandage compression only and dedicated radial artery compression devices may be used to achieve hemostasis in the novel approach. An important aspect of the use of this access may be a longer and tougher learning curve.

\section{Conflict of interest}

The authors declare no conflict of interest.

Corresponding author:

Łukasz Koziński MD, Department of Cardiology, District Hospital, 10 Lesna St, 89-600 Chojnice, Poland, phone: +48 506293497 ,

e-mail: lukekozinski@gmail.com

Received: 6.09.2018, accepted: 25.11.2018. 

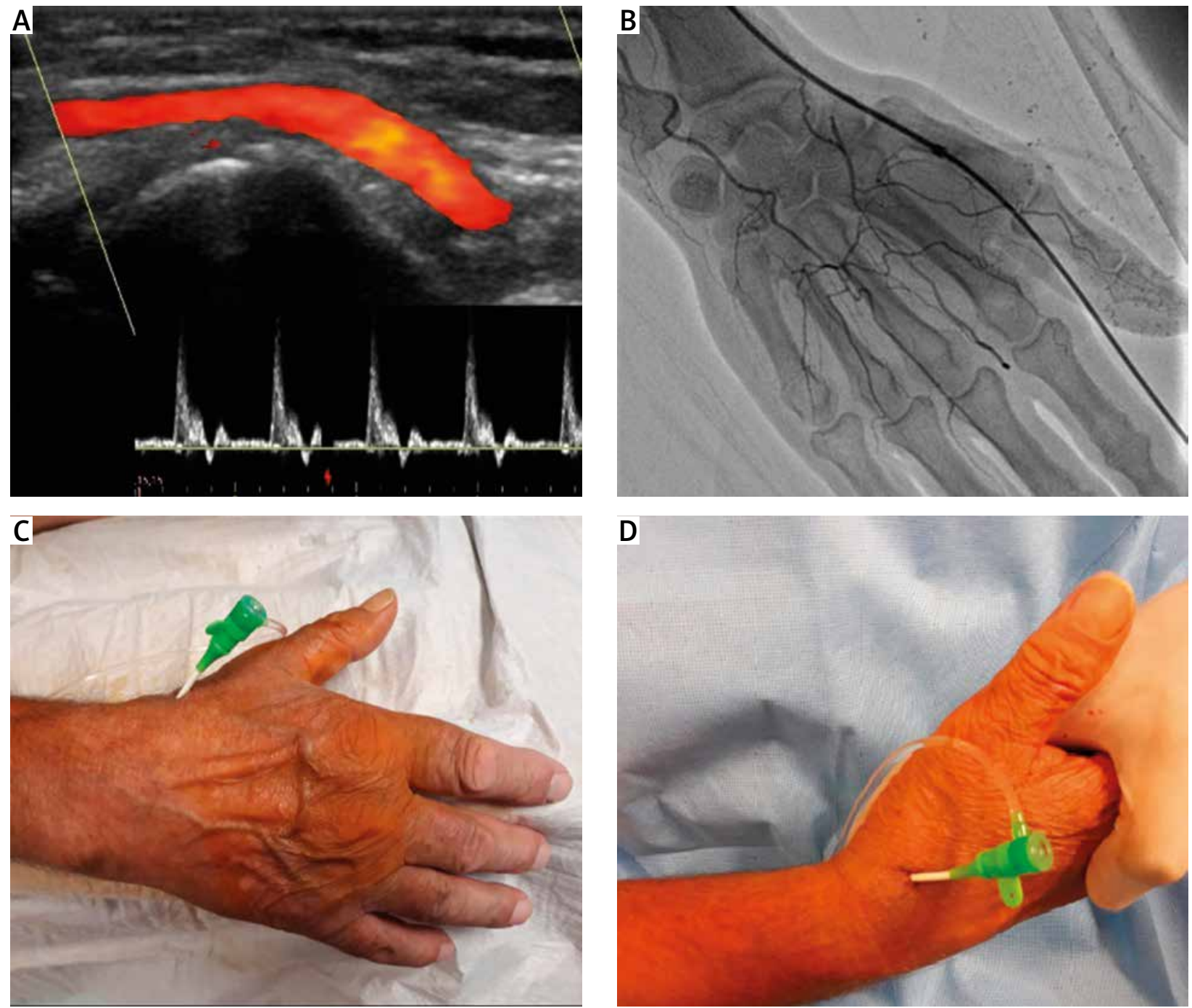

Figure 1. A - Preprocedural duplex ultrasonography of distal radial artery in anatomical snuffbox. B - Angiography of a hand's arterial circulatory after sheath insertion in a distal radial artery. C, D - A 6 Fr sheath inserted in the distal radial artery in the area of the anatomical snuffbox

\section{References}

1. Kiemeneij F. Left distal transradial access in the anatomical snuffbox for coronary angiography (IdTRA) and interventions (IdTRI). Eurolntervention 2017; 13: 851-7.

2. Soydan E, Akın M. Coronary angiography using the left distal radial approach - an alternative site to conventional radial coronary angiography. Anatol J Cardiol 2018; 19: 243-8.

3. Valsecchi O, Vassileva A, Cereda AF, et al. Early clinical experience with right and left distal transradial access in the anatomical snuffbox in 52 consecutive patients. J Invasive Cardiol 2018; 30: 218-23.

4. Andrade PB, Tebet MA, Rinaldi FS, et al. Initial experience with left distal transradial access for invasive coronary procedures. J Transcat Intervent 2018; 26: 1-5. 